of myocardial infarction, ${ }^{11} 12$ together with the relationship between the incidence of PVF and early admission in the hospital series, ${ }^{7}$ suggests electrophysiological disturbance within the heart leading to ventricular fibrillation but clearing within 24 hours.

Thus, although it is true that the heart at 17-18 hours after the onset of symptoms may not be especially vulnerable to serious arrhythmias and that they do not at this stage constitute an independent risk factor, the same conclusion cannot be drawn when these serious arrythmias occur within the first two hours.

University College Hospital,
London WC1E 6AU

Alan A Morgan

${ }^{1}$ McVeilly, R H, and Pemberton, J, British Medical fournal, 1968, 3, 139 .

2 Adgey, A A J, et al, Lancet, 1969, 1, 1169. 2,1169.

British Medical fournal, 1975, 1, 475.

Mitchell, J R A, and Schwartz, C J, Arterial Disease. Oxford, Blackwell, 1965.

6 Schwartz, C J, Atherosclerosis, 1972, 15, 1.

Lie, K I, Wellens, H J, and Durrer, D, European fournal of Cardiology, 1974, 1/4, 379.

Schwartz, C J, and Walsh, W J, Progress in Cardio-

vascular Diseases, 1971, 13, 465

Surawicz, 268.

Surawicz, B, Gettes, L S, and Ponce-Zumino, A, American fournal of Physiology, 1967, 212, 1519.

fourly, R H, and Pemberton, J, British Medical

Fournal, 1968, 3, 139 1969, 39-40, suppl No 4, p 182.

SIR,-I was very interested to read the article by Dr J M Roland and others (1 September, $p$ 518) on the effect of beta-blockers on arrhythmias during six weeks after suspected myocardial infarction. I would be in general agreement with their conclusion that betablockers in the dose used by them did not materially influence arrhythmias.

I fail to understand, however, why they concluded from their work that so-called serious and less serious ventricular arrhythmias have no part to play in the aetiology of ventricular fibrillation or death. Ninety-three patients were studied after definite or probable myocardial infarction and, as expected, the incidence of arrhythmias fell and became equal to that in non-infarct patients during follow-up. Hence "serious" arrhythmias are related to recent definite or probable myocardial infarct. We all know that it is patients with recent myocardial infarction who develop ventricular fibrillation, with decreasing frequency related to time after onset. It should, however, be noted that ventricular arrhythmia, even in these groups of patients, while occurring in a large percentage appears only infrequently in most patients. In this study (although their recordings did not start until 17 hours after onset of symptoms) 25 of the 49 episodes of ventricular tachycardia occurred in only one of the 31 patients taking placebo, implying that there were only 24 episodes in the remaining 30 patients-presumably only one or less episode occurring during the period of recording in a given patient. Nobody has ever claimed that every single ventricular ectopic or $\mathrm{R}$-on- $\mathrm{T}$ phenomenon results in ventricular fibrillation.

As the authors themselves admit, the patients monitored in the coronary care unit in their study represented a good-risk group. In this group one would expect a mortality of approximately $1 \%$ if haemodynamically they belonged to the group with a cardiac index of more than 2 litres a minute and a left ventricular end-diastolic pressure of less than $18 \mathrm{~mm} \mathrm{Hg}$ as one must assume most of their patients did. No conclusions can therefore be drawn from five deaths, three of which were in patients who had had serious arrhythmias recorded; nor can any conclusions be drawn from one patient with ventricular fibrillation who did not die and did not have any serious arrhythmia recorded, since after all the number of episodes in a given patient was infrequent during the recorded period and it is well known that arrhythmias vary in frequency from time to time.

Since the number of arrhythmias in a given patient was infrequent, conclusions regarding the efficiency or otherwise of betablockers are unsafe. There are a number of papers which suggest that patients differ in their response to a given dose.

\section{Murtaza Husaini}

General Hospital,

Ashton-under-Lyne, Lancs 0L6 9RW

\section{Dangerous antihypertensive treatment}

SIR,-Beta-blockers and diuretics act by influencing different controlling systems in the body, but Drs O W Barritt and A J Marshall may have given the impression in their recent letter ( 8 September, p 606) that the hypotensive effects of the two agents in combination may be predicted by summing the falls in blood pressure achieved with the individual drugs. This is not the case.

In a study by Castenfors and Danielsson 25 patients were given placebo for four weeks and then allocated to treatment with either $200 \mathrm{mg}$ metoprolol or $25 \mathrm{mg}$ hydrochlorothiazide for six weeks. They were then transferred to treatment with two tablets of Co-Betaloc, (a fixed combination of metoprolol, $100 \mathrm{mg}$, and hydrochlorothiazide, $12.5 \mathrm{mg}$ ) for a further six weeks. It was found that the blood pressure reduction, compared with placebo, was equivalent with metoprolol and hydrochlorothiazide $(16 / 8$ and $14 / 8$ $\mathrm{mm} \mathrm{Hg}$ ), although the number of patients satisfactorily controlled was somewhat higher in the metoprolol group. When Co-Betaloc was given a further reduction in blood pressure was achieved in both groups $(22 / 15 \mathrm{~mm} \mathrm{Hg}$ compared with placebo), but the fall obtained with combination therapy was appreciably less than the theoretical maximum (30/16 $\mathrm{mm} \mathrm{Hg}$ ) deduced from adding the effects of the individual drugs.

Drs Barritt and Marshall also imply that the use of beta-blockers and diuretics in combination doubles the chances of producing unwanted side effects. On the contrary, the use of high-dose monotherapy often leads to dose-related side effects which may be minimised by the use of a fixed low-dose combination product. This was illustrated in a recently completed multicentre hospital trial with Co-Betaloc (to be published), in which 140 hypertensive patients were treated for eight weeks. In this study only two patients were withdrawn owing to side effects. One patient complained of cold extremities, which was not surprising in view of the fact that the study ran from October to April during the coldest winter for over a decade. The other patient experienced profound muscular weakness resulting from a fall in serum potassium concentration from 4.3 to $3.0 \mathrm{mmol}(\mathrm{mEq}) / 1$. However, it transpired that this patient was also suffering from chronic glomerulonephritis, and the symptoms of weakness disappeared when the patient was transferred to a combination of metoprolol and the potassiumsparing diuretic amiloride.

Although one must be aware that combination therapy implies less flexibility and the consequent risk of over-treatment in patients who could have been satisfactorily treated with one of the components, this study indicates that a relatively low dose of hydrochlorothiazide in combination with metoprolol, given once a day, constitutes a simple, effective, and well-tolerated treatment for patients with essential hypertension.

RICHARD GOODFELLOW

Astra Pharmaceuticals Ltd

St Albans, Herts AL1 3JW

' Castenfors, J, and Danielsson, M, Current Therapeutic Research, 1979, 25, 228.

\section{Routine exercise testing in angina} pectoris

SIR,-Dr J A Raffo and others (4 August, p 295) have reported on the diagnostic value of routine exercise testing in hospital patients with angina pectoris. They claimed that their method "predicted the existence of radiologically demonstrable lesions with greater accuracy than other reported methods" and that "this improvement was achieved merely by carefully selecting the patients."

In their study 132 selected patients with angina pectoris underwent exercise testing on a bicycle ergometer. The results of tests on 32 patients were discarded because heart rate did not increase to $85 \%$ of the predicted or there was a lack of agreement in interpretation of the record. The results from the remaining 100 patients indicated that the response to exercise correlated well with abnormalities of the coronary arteries detected by angiography. Although this establishes the significance of an adequately performed exercise test, a fairer assessment of the clinical value of the test should include the 32 patients who were excluded. The results would then be less favourable.

We have assessed exercise testing in a similar group of patients but with a maximal treadmill test and a 12-lead recording system for the electrocardiogram. ${ }^{1}$ Our study and those of others ${ }^{2-4}$ show that in patients with angina pectoris the sensitivity of the multilead exercise test is greater than that of single-lead systems. The use of multiple leads also has the advantages that the site of ischaemia can be localised and that multi-vessel disease is associated with abnormalities in a greater number of leads ${ }^{12}$ and with the sum of the ST segments in all leads. ${ }^{1}$ This additional information is important since coronary artery bypass surgery may prolong life in patients with disease of all three coronary arteries or the left main coronary artery.

A 12-lead electrocardiogram during and after a treadmill exercise test is more expensive than a single-lead test because of the need for a three-channel recorder, the extra time needed to attach the electrodes, and the price of electrodes ( $£ 1.50$ per patient). This cost is, however, relatively trivial when compared with that of cardiac catheterisation and coronary angiography. While agreeing with Dr Raffo and his colleagues that exercise testing is useful in the management of patients with angina pectoris, we believe that the additional information obtained from a 